EPJ Web of Conferences 73, 05005 (2014)

DOI: $10.1051 /$ epjconf/20147305005

(C) Owned by the authors, published by EDP Sciences, 2014

\title{
Chiral symmetry restoration in $\sigma$-meson production in hadronic processes
}

\author{
V.I. Kukulin ${ }^{\mathrm{a}}$ and M.N. Platonova \\ Skobeltsyn Institute of Nuclear Physics, Lomonosov Moscow State University, Moscow 119991, \\ Russia
}

\begin{abstract}
Some puzzles about the nature and properties of the lightest scalar meson, $\sigma$ or $f_{0}(500)$, are analyzed in the paper. We studied the $\sigma$-meson production both in $N+N$, $N+d$, etc., collisions and also in $J / \psi, \psi(2 S), \psi(3 S), \Upsilon(2 S)$, etc., two-pion decays. The fundamental distinctions between the basic $\sigma$-meson parameters found in various hadronic processes can be explained most naturally by the chiral symmetry restoration in intermediate excited hadronic resonances. In the present paper we discuss some important aspects of chiral symmetry restoration in hadronic processes with interrelation to the basic features of QCD.
\end{abstract}

\section{Introduction. The riddle of $\sigma$ meson}

Initially the $\sigma$ particle, the lightest scalar-isoscalar meson, was predicted by Schwinger [1] and also by Gell-Mann and Levy [2] (within their linear $\sigma$ model) to exist as a chiral partner of the NambuGoldstone $\pi$ meson, corresponding to the dynamical breaking of chiral symmetry (conserving in the massless limit of QCD), with the mass $m_{\sigma} \simeq 2 m_{q}, m_{q}$ being the constituent quark mass. This $\sigma$ meson gives the constituent quark masses and thus it plays the role of the Higgs particle in QCD. From the other hand, the $\sigma$ meson was predicted to play a key role in nuclear force at intermediate distances $r_{N N} \leq 1 \mathrm{fm}$. According to the conventional theory of nuclear force [3], just the above light scalar meson exchange between two nucleons should be responsible for the strong $N N$ attraction at $r_{N N} \leq 1 \mathrm{fm}$, if such scalar meson exists. However, from the $\pi \pi$ phase-shift analysis derived from CERN-Munich experiment of 1974 [4] one observed a rather smooth behavior of the $\delta_{0}^{0}$ phase in $\pi \pi$ scattering up to invariant mass $M_{\pi \pi}=1100 \mathrm{MeV}$ which seems hardly compatible with a well defined scalar resonance. As a result, the light $\sigma$ particle disappeared from the list of PDG since 1976 edition for more than 20 years! And the direct $t$-channel $\sigma$ exchange also disappeared from the current theories of nuclear forces.

Nevertheless, in many old and recent experiments one still observes a very large event concentration in the $I=0 s$-wave $\pi \pi$ channel which cannot be explained as a simple "background" and seems to strongly suggest the existence of $\sigma$. A huge accumulation of the low-mass $\pi \pi$ events in high-energy $p p$ collisions and $J / \psi \rightarrow \omega \pi \pi$ decay are demonstrated in Figs. $1 a$ and $b$ respectively [5]. A further

\footnotetext{
ae-mail: kukulin@nucl-th.sinp.msu.ru
}

This is an Open Access article distributed under the terms of the Creative Commons Attribution License 4.0, which permits unrestricted use, distribution, and reproduction in any medium, provided the original work is properly cited. 

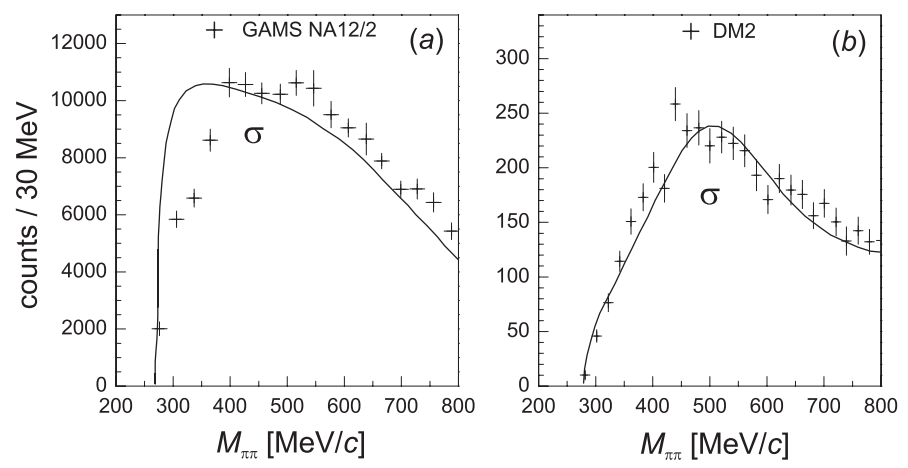

Figure 1. Phenomenological observation of the $\sigma$ meson in production processes: $(a) \pi^{0} \pi^{0}$ invariant-mass distribution in $p p$ central collisions (GAMS NA12/2) and (b) $\pi \pi$ invariant-mass distribution in $J / \psi \rightarrow \omega \pi \pi$ decay (DM2).

significant strengthening for the existence of the light $\sigma$ meson has been found in numerous hadron spectroscopy experiments [6]. Today one observes very numerous data from the charmonium $\psi(2 S)$, $\psi(3 S)$, etc., and bottomonium $\Upsilon(2 S), \Upsilon(3 S)$, etc., decays leading to strong yield of light scalar mesons with the mass in range $m_{\sigma} \simeq 450-550 \mathrm{MeV}$, and the width $\Gamma_{\sigma} \simeq 350-450 \mathrm{MeV}$. Most of the respective data have been collected in BESII and BESIII Collaborations experiments on $e^{+} e^{-}$collisions (see, e.g., [7]).

Quite recently, by using the subtracted $\pi \pi$ dispersion relations, a rather accurate pole position for the $\sigma$ meson have been derived [8]: $m_{\sigma}^{(\pi \pi)}=441_{-8}^{+16} \mathrm{MeV}, \Gamma_{\sigma}^{(\pi \pi)}=544_{-25}^{+18} \mathrm{MeV}$. So, all the values obtained for $m_{\sigma}$ and $\Gamma_{\sigma}$ from different experiments and theoretical approaches are in a general agreement with each other, and also with the $\pi \pi$ PSA data. However, it should be stressed that the scalar meson with the huge width $\Gamma_{\sigma} \sim 500 \mathrm{MeV}$ is so short-lived that it is absolutely unable to carry a strong intermediaterange attraction between two nucleons postulated in traditional OBE-like models of nuclear force, because its free path is only $\lambda_{\sigma}=c \hbar / \Gamma_{\sigma} \simeq 0.2 \mathrm{fm}$ !

On the other hand, if to assume the formation at short $N N$ distances of a unified six-quark bag dressed with various meson fields, the dominant contribution to a meson cloud of the bag comes just from the scalar $\sigma$ meson due to its very strong attraction to the $6 q$ core. But this is only beginning of the long story, because the contribution of the $\sigma$ loops to the intermediate dressed bag (dibaryon) propagator can be negligible if the $\sigma$ width is as large as $500 \mathrm{MeV}$. So that, in order to get a significant contribution from the $\sigma$-meson cloud to the dibaryon propagator, the $\sigma$ width should be much lower.

\section{Dibaryon model for short-range nuclear force and chiral symmetry restoration in excited hadrons}

The dibaryon mechanism looks to be ideally suited to describe the short-range $N N$ force. It is because the mechanism assumes generation of the intermediate "long-lived" quark-meson states and such a resonance-like state will enhance somehow the short-range $N N$ interaction. The particular mechanism for the dressed dibaryon formation at short $N N$ distances (proposed still in 1998 [9]) is as follows:

$$
N+N \rightarrow\left|s^{4} p^{2}[42] L_{q}=0,2 ; S T\right\rangle \rightarrow\left|s^{6}[6] L_{q}=0 ; S T+\sigma\right\rangle .
$$

The corresponding graph for short-range $N N$ interaction in Born approximation is depicted in Fig. 2a. The total $\sigma$-dressed dibaryon propagator should be of course a sum of the $\sigma$ loops (see Fig. 2b).

In accordance with general rules for the Feynman graphs, the mechanism presented in Fig. 2a can be described by a separable potential $V_{N q N}=\lambda(E) g(\mathbf{p}) g\left(\mathbf{p}^{\prime}\right)$. The form factor $g(\mathbf{p})$ corresponds 


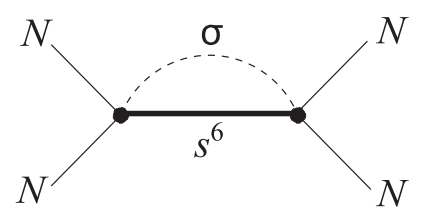

(a)

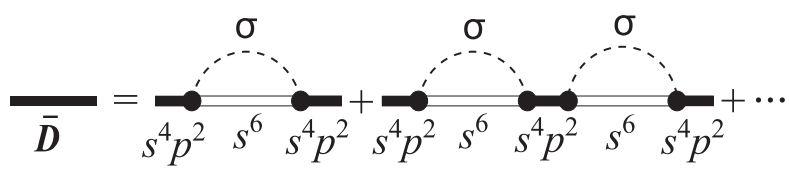

(b)

Figure 2. (a) $N N$ potential corresponding to the $s$-channel $\sigma$-dressed dibaryon exchange (the Born term). (b) The $\sigma$ dressing of the intermediate dibaryon propagator.

to a transition vertex from the initial $N N$ to the mixed-symmetry $6 q$ state, i.e., $N N \rightarrow\left|s^{4} p^{2}[42]\right\rangle$, and the energy-dependent coupling constant $\lambda(E)$ corresponds to the intermediate dressed dibaryon propagation: $\lambda(E)=\int d^{3} k B^{*}(\mathbf{k}) B(\mathbf{k}) /\left(E-M_{D}-k^{2} / 2 M_{D}-\varepsilon_{\sigma}(k)\right)$, where $B(\mathbf{k})$ stands for the $\sigma$ production vertex $\left|s^{4} p^{2}[42]\right\rangle \rightarrow\left|s^{6}[6]+\sigma\right\rangle$. Thus, to calculate the short-range $N N$ potential one has to know only some basic parameters of the dressed six-quark bag (the mass and radius of the intermediate dibaryon) [10]. In case of two channels ${ }^{3} S_{1}-{ }^{3} D_{1}$ coupled by a short-range tensor force (which is originated, e.g., from the one-gluon exchange among six quarks in the bag) one gets the two-channel separable potential (for non-relativistic case) [10]. The consistent relativistic generalization of the above dibaryon model in terms of the effective field theory has been developed in Ref. [11].

It should be stressed that the above dibaryon mechanism with the $\sigma$ loops can be effective only if the mass of the intermediate dibaryon dressed with the $\sigma$-meson cloud is rather low $\left(M_{D} \simeq 2.3-\right.$ $2.5 \mathrm{GeV}$ ), otherwise the coupling constant $\lambda(E)$ and hence the probability of the dibaryon generation will be insufficient to provide the intermediate-range $N N$ attraction. To our fortunate, there is a very important QCD mechanism, that is, the Chiral Symmetry Restoration $(\chi \mathrm{SR})$, which was shown to play an essential role in highly excited hadrons [12]. In particular, the (partial) $\chi S R$ effects lead to appearance of (approximate) parity doublets in hadronic spectra, i.e., the excited states with opposite parities but with the same spins are degenerated. The physical origin of the $\chi \mathrm{SR}$ phenomenon is rather simple: when the quark kinetic energy inside a hadron is rising, the gluon (quark-antiquark) condensate is diminishing (it gets "uncoupled" from valence quarks) and thus the quark mass goes down to the bare (current) one. The recent theoretical investigations based on the Dyson-Schwinger equations as well as the latticeQCD calculations show this fundamental result very clearly [13].

Due to the (partial) $\chi$ SR effect the masses of a six-quark bag (which is a $2 \hbar \omega$-excited $6 q$ state in the dibaryon model) and of the $\sigma$ mesons surrounding the bag get renormalized strongly, i.e., $M_{D} \rightarrow 2.2 \mathrm{GeV}$ and $m_{\sigma} \rightarrow 300 \mathrm{MeV}$. It is important that the pion mass remains almost constant within the chiral transition, so the width for the $\sigma \rightarrow \pi \pi$ decay gets strongly reduced. Thus, the $\sigma$ field surrounding the $6 q$ bag gets much more stable in comparison with the "free" $\sigma$ field. In such circumstances, the dressed dibaryon formation can occur with a sufficient probability to yield the effective intermediate-range $N N$ attraction [14]. Thus, we can conclude that dibaryon plays a role of a catalyst for the $\sigma$-meson production in $N N$ collisions at intermediate energies.

\section{Novel treatment for the $A B C$ effect in $2 \pi$ production}

The ABC effect [15] stands for a strong enhancement of $2 \pi$ production very near to the $2 \pi$ threshold in the scalar-isoscalar channel, i.e., $\pi^{0} \pi^{0}$ or $\left(\pi^{+} \pi^{-}\right)_{0}$, in $p+n, p+d$ and $d+d$ fusion reactions. In most theoretical works done in this field for the passed 50 years the effect was explained by the nearby $\Delta \Delta$ threshold. However, the new exclusive experimental data of WASA-at-COSY Collaboration [16] occurred to be incompatible with such a model. The experimentalists have found a very clear signal of the dibaryon resonance formation in $2 \pi$-production cross section in $p+n$ collisions at $T_{p} \simeq 1-1.4 \mathrm{GeV}$. The following resonance parameters were extracted from the experimental data [16]: $I\left(J^{P}\right)=0\left(3^{+}\right)$, 


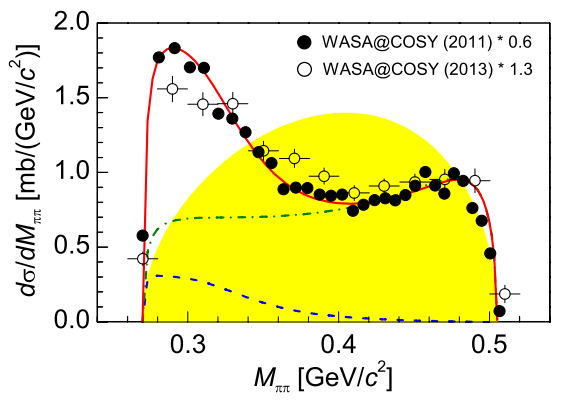

Figure 3. The $\pi \pi$ invariant-mass spectrum in the reaction $p n \rightarrow d+\pi^{0} \pi^{0}$ at the energy $\sqrt{s}=2.38 \mathrm{GeV}$. The results of the dibaryon model calculations (solid line), the contributions of $\sigma$-meson production (dashed line) and $D_{12}$-dibaryon generation (dash-dotted line) mechanisms and the pure phase-space distribution (shaded area) are shown. Experimental data (open and filled circles) are taken from Refs. [16] and renormalized to the experimental value of the total cross section $\sigma_{t}=0.25 \mathrm{mb}$.

$M_{R} \simeq 2.37 \mathrm{GeV}$ and $\Gamma_{R} \simeq 70 \mathrm{MeV}$. Furthermore, the ABC effect was clearly identified with $2 \pi$ emission from this dibaryon state, but the dynamical mechanism of this emission remained unclear.

Recently we reanalyzed the new WASA-at-COSY experimental data in terms of the dibaryon model [17, 18]. It was suggested [17] that the strong ABC peak in the $\pi \pi$ invariant-mass spectrum in scalar-isoscalar channel and an almost isotropic angular distribution of two pions in their c.m. frame in the region of the peak can be naturally explained by the intermediate $\sigma$-meson production originated from the excited dibaryon. Our model includes also a mechanism for sequential $2 \pi$ production via an intermediate isovector dibaryon with $I\left(J^{P}\right)=1\left(2^{+}\right)$(denoted by $D_{12}$ ). By adding coherently the above two amplitudes, we obtained a very good description of the various experimental data with only a few model parameters [17]. The results of the dibaryon model calculations for the $\pi \pi$ invariant-mass distribution in the basic reaction $p n \rightarrow d+\pi^{0} \pi^{0}$, i.e.,

$$
d \sigma / d M_{\pi \pi}=(\text { phase space }) \times \iint d \Omega_{d}^{c . m} \cdot d \Omega_{\pi}^{\pi \pi} \sum_{\text {spins }}\left|A^{(\sigma)}+A^{\left(D_{12}\right)}\right|^{2},
$$

are shown in Fig. 3. The best fit of the data (filled circles in Fig. 3) was achieved in the dibaryon model with the $\sigma$-meson mass $m_{\sigma} \simeq 300 \mathrm{MeV}$ and width $\Gamma_{\sigma} \simeq 100 \mathrm{MeV}$, which are strongly reduced as compared to their free-space values extracted from $\pi \pi$ dispersion relations [8]. Such a reduction for the $\sigma$-meson parameters can be understood as a direct signal of the partial $\chi \mathrm{SR}$ in the excited $0\left(3^{+}\right)$ dibaryon state whose excitation energy is $E^{*} \simeq 500 \mathrm{MeV}$. As was stated above, just the $\chi \mathrm{SR}$ effects in the intermediate ( $2 \hbar \omega$-excited) dibaryons, which drive the short-range nuclear force in the dibaryon model, can provide an effective $N N$ attraction at intermediate distances $r_{N N} \simeq 0.7-1 \mathrm{fm}$, due to the two basic effects: strengthening the coupling of the light $\sigma$ meson to the $6 q$ core and the mass renormalization of the $6 q$ core itself. This, in turn, demonstrates clearly the highly nontrivial mechanism which stands behind the short-range $N N$ interaction. So, we can summarize: the nature of short-range nuclear force is likely based on the mechanism of chiral symmetry restoration in the intermediate quark-meson state.

\section{References}

[1] J. Schwinger, Ann. Phys. 2, 407 (1957)

[2] M. Gell-Mann, M. Levy, Nuovo Cim. 16, 705 (1960)

[3] R. Machleidt, Adv. Nucl. Phys. 19, 189 (1989)

[4] G. Grayer et al., Nucl. Phys. B 75, 189 (1974) 
[5] S. Ishida, AIP Conf. Proc. 432, 705 (1998)

[6] T.A. Armstrong et al., Z. Phys. C 51, 34 (1991)

[7] M. Ablikim et al., Phys. Lett. B 645, 19 (2007)

[8] I. Caprini, G. Colangelo, H. Leutwyler, Phys. Rev. Lett. 96, 132001 (2006)

[9] V.I. Kukulin, in Proc. 5th PNPI School on Theoretical Physics, Gatchina, 1999, p. 142

[10] V.I. Kukulin, I.T. Obukhovsky, V.N. Pomerantsev, A. Faessler, Int. J. Mod. Phys. E 11, 1 (2002)

[11] A. Faessler, V.I. Kukulin, M.A. Shikhalev, Ann. Phys. (N.Y.) 320, 71 (2005)

[12] L.Ya. Glozman, Phys. Lett. B 475, 329 (2000)

[13] C.D. Roberts, Prog. Part. Nucl. Phys. 61, 50 (2008)

[14] V.I. Kukulin et al., Ann. Phys. (N.Y.) 325, 1173 (2010)

[15] N.E. Booth, A. Abashian, K.M. Crowe, Phys. Rev. Lett. 7, 35 (1961); 5, 258 (1960)

[16] P. Adlarson et al., Phys. Rev. Lett. 106, 242302 (2011); Phys. Lett. B 721, 229 (2013)

[17] M.N. Platonova, V.I. Kukulin, Phys. Rev. C 87, 025202 (2013)

[18] M.N. Platonova, Few-Body Syst. doi:10.1007/s00601-013-0757-3 (2013) 